This manuscript is a non-peer reviewed preprint submitted to EarthArXiv. Subsequent versions of this manuscript may have slightly different content. Please feel free to contact the corresponding author Omid Dorostkar at domid@ethz.ch for feedback. 


\title{
On the effect of grain fragmentation on frictional instabilities in faults with granular gouge
}

\author{
Di Wang ${ }^{1,2}$, Jan Carmeliet $^{1}$, Wei Zhou ${ }^{2 *}$, Omid Dorostkar ${ }^{1,3^{*}}$
}

1. Department of Mechanical Engineering, Swiss Federal Institute of Technology Zurich (ETH Zurich), Zurich, Switzerland

2. State Key Laboratory of Water Resources and Hydropower Engineering Science, Wuhan University, Wuhan, China

3. Department of Engineering Science, University of Oxford, Oxfordshire, Oxford, UK

*Corresponding authors: Omid Dorostkar(domid@ethz.ch), Wei Zhou (zw_mxx@whu.edu.cn)

\begin{abstract}
The evolution of frictional strength during stick-slip dynamics of a fault system is key to understanding the earthquake nucleation and rupture patterns. In mature faults, granular gouge is produced by wear, comminution or fragmentation during tectonic movements. In this work, we introduce a fragmentation model in the simulation of a sheared granular fault to explore the influence of grain breakage on the stick-slip dynamics. With fragmentation of highly stressed particles, the fault frictional strength increases accompanied with many small slip events triggered by particle breakage. The small fragments produced by particle breakage are not only stronger and more difficult to break, but they also change the distribution of contact forces, leading to strengthening of the fault system. By statistical analyses on size distribution of slip events under different particle strengths, we find that when particles are weaker, slip events become more correlated with particle fragmentation events and that the number of large slip events decreases. In addition, our analyses on the relationship between slip and particle fragmentation events reveal three types of correlations: in the first and second types, particle fragmentation events trigger micro- or major slips, respectively. In the third category, large-scale particle fragmentations take place at the end of large slip events owing to stress localization during post-slip particle rearrangements. Our results in this work highlight the role of micromechanics of particle fragmentation in failure of fault damage zones and help in understanding the relation between particle breakage and frictional failures.
\end{abstract}

Keywords: stick-slip dynamics, fault gouge, friction, particle fragmentation, comminution 


\section{1- Introduction}

Mature faults contain granular gouge materials that are usually created by wear, fragmentation, and comminution of host rock due to tectonic loading (Engelder, 1974; Billi et al., 2003; Collettini et al., 2009). It is shown that the frictional stability and properties of gouge materials affect the sliding behaviour of the fault system, playing a key role in the earthquake nucleation and rupture processes (Scholz et al., 1972; Scholz, 1998; Scuderi et al., 2017; Leeman, 2016). The physics of earthquake nucleation and fault slip have been widely explored through scaled stick-slip experiments and numerical simulations, providing insights into the role of loading configuration, grain properties, and the physicochemical interactions on the stability of fault system and the characteristics of stickslip cycles [e.g. (Mair et al., 2002; Jiang et al., 2016; Johnson et al., 2016; Dorostkar et al. 2017, 2018)]. In particular, at high confining stress, particles undergo fracture and fragmentation (Sammis et al., 1987; Blenkinsop, 1991; Storti et al., 2003; Billi, 2004, 2005; Davies et al., 2012). Naturally, fault comminution will lead to highly evolved fault gouge with a fractal distribution of particle size, which dramatically changes the frictional properties and permeability of fault (Storti et al., 2003). For other frictional instabilities like landslides, intensive grain fragmentation in force chains is found to dramatically reduce the effective stress of the granular flow and correspondingly decrease the frictional resistance to sliding (Davies and McSaveney, 2009). By analysis of the elastic strain energy in fault rupture, Davies et al. (2012) found grain fragmentation converts the energy between kinetic and potential energy. When a grain breaks and generates many new fragments, these fragments will gain unstrained shapes as much as is permitted by their new constraints and they will release their stored potential energy. A certain amount of potential energy transforms into surface energy and frictional heat that no longer participates in the future energy cycle (Griffith, 1920). The rest of the potential energy converts into kinetic energy of fragments if the grain is unconfined (Bergstrom et al. 1961; Davies et al. 2012), or, under confined conditions, transmits into elastic energy in the surrounding grains. If particle fragmentations occur in force chains, the released energy stems from both grain fragmentation and elastic unloading of other grains that applied the failure stress. The transmitted elastic energy may generate loading of other particles eventually leading to failure of force chains or even substantial breakages nearby (Davies and McSaveney, 2009).

To further understand the evolution of fault damage zone and the effect of commination, many laboratory studies were performed with simulated fault gouge. Marone and Scholz (1989) reproduced experimentally the fractal Particle Size Distribution (PSD) in the simulated fault layer and found significantly more comminution in sheared layers than the overall sample, indicating that the relative movement between particles dominates comminution. As fragmentation leads to both wider PSDs and change of particle shape, Mair et al. (2002) performed a series of experiments to address the influence of each factor and found that wider PSDs tend to be stable and reduce the stick-slip dynamics exhibited in narrower PSDs. Meanwhile, if the spherical particles break into angular fragments, the frictional strength of fault will increase significantly (Mair et al., 2002). In a different experimental setup, Jiang et al. (2017) compared the shear resistance of different sizes of silica sand and found smaller grains have higher peak and residual shear strength. If gouge particles are continuously crushed, generating very fine grains in fault, the fine particles will behave similarly to powder lubricants and dramatically weaken the strength of the fault (Reches and Lockner, 2010). 
As numerical modelling can track the mechanical interaction between particles and visualize dynamic processes of granular flow at grain scale, it has also been widely applied for studying fracture and fragmentation in fault systems. Abe and Mair (2005) enhanced the 3D lattice solid model and successfully reproduced the evolution of fractal PSD in fault gouge. They performed a series of simulations on exploring the grain size distribution, fragments shapes and the relationship between particle breakage and strain localization (Mair and Abe, 2008, 2011; Abe and Mair, 2009), and found that the frictional strength of fault gouge can be enhanced by a reduction in porosity and increasing angularity of fragments caused by grain breakage (Guo and Morgan et al., 2006; Mair and Abe, 2008; Abe and Mair, 2009). Under shear stress, the fragmentation of particles vary from major splitting at the initial stage leading to angular grains, to abrasion of particles at large shear strain decreasing the angularity of particles (Guo and Morgan et al., 2007; Mair and Abe, 2011). Therefore, the fragments may be of angular shape and strengthen the fault or, in the end, become round, fine grains that lubricate the fault.

There is, however, limited work focused on the relationship between the stick-slip dynamics as a key mechanism behind earthquakes and grain breakage in a sheared fault system. What remains unclear is the influence of particle breakage on the frictional stability of fault gouge, i.e., the statistical distribution of slip event size, the micromechanics of grain rearrangements and the stress distribution and localization before and after a series of particle fragmentation events. Our aim in this work is to model grain fragmentation and study its effect on the characteristics of stick-slip cycles. We perform statistical analyses on the size distribution of slip events in long simulations and evaluate the effect of grain strength on slip size and the recurrence time. Using micromechanical metrics, we also look into the grain-scale mechanisms underlying the macroscopic observations. Our simulations provide new insight into the relationship between grain fragmentation and shear behaviour of fault gouge, which is significant in understanding the frictional stability of fault systems.

\section{2- Method}

The granular fault gouge model has a sample size of $11 \times 1.5 \times 0.8 \mathrm{~mm}^{3}$ with periodical boundaries across $\mathrm{x}$-direction and frictionless walls in y-direction (Fig. 1a). Confining stress is applied by two corrugated plates in the z-direction. The granular layer consists of particles with a diameter ranging 90-150 $\mu \mathrm{m}$ with a uniform distribution. After confinement to a given normal stress, the bottom plate moves in the x-direction with a constant velocity (shear rate). A summary of the DEM parameters i.e. confining stress, shear rate, as well as number, size and properties of the particles and time step is listed in Table 1, which are mostly based on our previous works (Dorostkar and Carmeliet, 2018, 2019). However, in order to show the long-term influence of particle fragmentation, a higher shear rate of $12 \mathrm{~mm} / \mathrm{s}$ is applied to obtain more particle fragmentation events.

We use the discrete element method (DEM) combined with a particle fragmentation model (fragment replacement model) to investigate the stick-slip dynamics in a granular fault gouge. In the particle breakage model, each particle has a strength representing the pressure endurance, where this strength is compared with the particle's stress. If the particle octahedral shear stress exceeds the 
particle strength, it will be crushed and replaced with several smaller spherical fragments. This simplified but efficient model is widely used to capture the effect of fragmentation in granular systems. An advantage of this approach compared to those where fragmentation is modelled by breaking bonds between particles of which grains are constituted, is that our approach minimizes the number of particles in the simulation.

The strength of soil particles is widely accepted to be statistically described by a Weibull type distribution as follows (McDowell and Bolton 1998; Zhou et al., 2016):

$$
P_{s}(d)=\exp \left(-\left(\frac{d}{d_{0}}\right)^{3}\left(\frac{q_{0}}{q_{\text {crit }}}\right)^{m}\right)
$$

where the stress $q_{\text {crit }}$ is the critical stress for a specimen of characteristic particle size $d_{0}$ such that $37 \%$ of tested specimens survive. The variable $q_{0}$ is the individual particle strength and $m$ is the Weibull modulus, which increases as the variation in strength decreases. Because larger particles show a higher probability of having more and larger flaws or cracks, a size-weakening law is considered as $-\left(\frac{d}{d_{0}}\right)^{3}$ in Eq. (1), meaning that larger particles are weaker.

Fragmentation of particles is controlled by a breakage criterion using the octahedral shear stress $q$ as proposed by McDowell and de Bono (2013) and defined as:

$$
q=\frac{1}{3}\left[\left(\sigma_{1}-\sigma_{2}\right)^{2}+\left(\sigma_{2}-\sigma_{3}\right)^{2}+\left(\sigma_{1}-\sigma_{3}\right)^{2}\right]^{0.5},
$$

where $\sigma_{1}, \sigma_{2}$ and $\sigma_{3}$ are the principal stresses. The stress tensor of an individual particle is defined as (McDowell and de Bono, 2013):

$$
\sigma_{i j}=\frac{1}{V} \sum_{N_{c}}\left(x_{i}^{c}-x_{j}^{p}\right) F_{i}^{c, p},
$$

where $V$ is the particle volume, $N_{c}$ is the number of contacts for the particle, $x_{i}^{c}$ and $x_{j}^{p}$ are the position of the contact and particle for any particle $\mathrm{i}$ and its neighbouring particle $\mathrm{j}$, and $F_{i}^{c, p}$ is the contact force at contact point $c$ acting on particle i. Once the octahedral shear stress surpasses the particle strength $q_{0}$, the particle is assumed to fragment.

With respect to particle breakage into several fragments, Marketos and Bolton (2009) used a simplified replacement method in the simulation of compaction band to observe post-localization crushing behaviour near the band. McDowell (2013) also used this model for oedometer test simulations, reproducing the normal compression line consistent with theoretical predictions. Ciantia (2015) made an overall validation of this model by comparing the normal compression line, grain size distribution, lateral pressure coefficient and computational burden with reported experimental data. Recently, this model has been successfully applied for exploring the fractal micromechanics of particle crushing in granular materials under normal compression (McDowell, 
In the above fragmentation model, once the particle stress exceeds the particle strength, the particle is replaced with several spherical fragments following a certain replacement pattern. While different replacement models have been developed based on experimental observations (Zhou et al., 2020), to capture the major influence of breakage and neglect the effect of many small debris, replacement models only consider the splitting of a particle into several larger progeny particles (McDowell and de Bono, 2013; Zhou et al., 2015). On the other hand, researchers have stressed the important role of small debris in granular flows and proposed substantial crushing mode, which includes both major fragments and some fine debris (Marketos and Bolton, 2009; Zhou et al., 2016). Ciantia et al. (2015) used apollonian sphere packing, which includes the splitting into both large and smaller particles consistent with the fractal grain size distribution of fragments. In our work and in order to keep the simulation computationally efficient, we choose the four-particles apollonian sphere as replacement model. In this approach, a broken particle is replaced with four equal size particles with arrangement of tangent spheres within the spherical space (Fig. 1b). When a particle is fragmented, the replacing particles do not equal to the volume of the original particle, leading to mass loss. To address this problem, Ciantia et al. (2015) assumed that the mass loss is stemmed from finer particles that contribute very little to the macroscopic mechanical response of the system and simulated the particle breakage without considering accurate mass conservation. Alternatively, McDowell et al. (2013) used a replacement model with overlapping particles to conserve mass. However, this approach introduces artificially additional energy into the system. To conserve both mass and energy, we apply a new strategy to the four-particles apollonian model: firstly, four ghost particles are inserted into the spherical space of broken particle with apollonian sphere packing (see Fig. 1c-d). Then we expand the radius of ghost particles to retain the original mass. So far, the mass conservation strategy is the same as in our previous work (Zhou et al., 2020), except that the ghost particles are assumed not to interact with the other particles in the simulation. Next, the fragments are moved opposite to the overlap region providing a small random spatial disturbance (Fig. 1e). Due to this disturbance, the fragments may overlap with other particles. These particles are again moved opposite to the overlap. This fragment adjustment procedure is repeated until the particles do not overlap anymore reaching a new configuration of particle fragments (Fig. 1f).

We slide the bottom corrugated plate (Fig. 1a) under constant confinement stress until the granular gouge reaches its steady-state macroscopic friction and stick-slip dynamics commences. We then turn on the fragmentation model using a given particle strength. The octahedral shear stress is calculated every 5000 time steps and particles with an octahedral shear stress that reaches particle strength are replaced with their respective fragments. As fragmentation leads to a reduction of average particle size, we also decrease the time step to maintain the simulation stable. Since in reality, fragmented particles have higher angularity and roughness (Mair et al. 2002), we also increase the inter-particle friction for replacing particles to represent the different roughness between spherical beads and their spherical fragments. The critical particle strength $\left(q_{\text {crit }}\right)$ is taken from a single particle crushing test and is found ranging from 110 to $147 \mathrm{MPa}$, depending on the characteristic particle size (Nakata et al., 2001). We found that this strength is too high to allow sufficient particles to fragment under a confining stress of $10 \mathrm{MPa}$. Therefore, we perform a sensitivity analysis by lowering the critical strength systematically allowing the number of 
fragmented particles to grow from tens to thousands (in our simulation we have initially around 8000 particles). With this approach, from one hand we control our computational cost but also represent particle characteristics as close as possible to laboratory experiments. Our DEM model is developed based on the open source software LIGGGHTS (Kloss et al. 2012) as discussed extensively in our previous works (Dorostkar et al., 2017a, 2017b, 2018; Dorostkar and Carmeliet, 2018, 2019; Ren et al., 2019; Zhou et al., 2020).

Table 1

\begin{tabular}{lclc}
\hline Property & Value & Property & Value \\
\hline Confining stress & $10 \mathrm{MPa}$ & Sample size & $11 \times 1.5 \times 0.8 \mathrm{~mm}^{3}$ \\
\hline Shear rate & $12 \mathrm{~mm} / \mathrm{s}$ & Particle diameter & $90-150 \mu \mathrm{m}$ \\
\hline Density & $2900 \mathrm{~kg} / \mathrm{m}^{3}$ & Number of particles & $7996-16715$ \\
\hline Poisson ratio & 0.25 & Particle friction coefficient & $0.1-0.9$ \\
\hline Young's modulus & $65 \mathrm{GPa}$ & Particle restitution coefficient & 0.87 \\
\hline Critical strength & $25 \mathrm{MPa}-\infty$ & Weibull modulus & 2.17 \\
\hline Characteristic particle size & $250 \mu \mathrm{m}$ & DEM time step & $0.5-1.5 \times 10^{-8} \mathrm{~s}$ \\
\hline
\end{tabular}

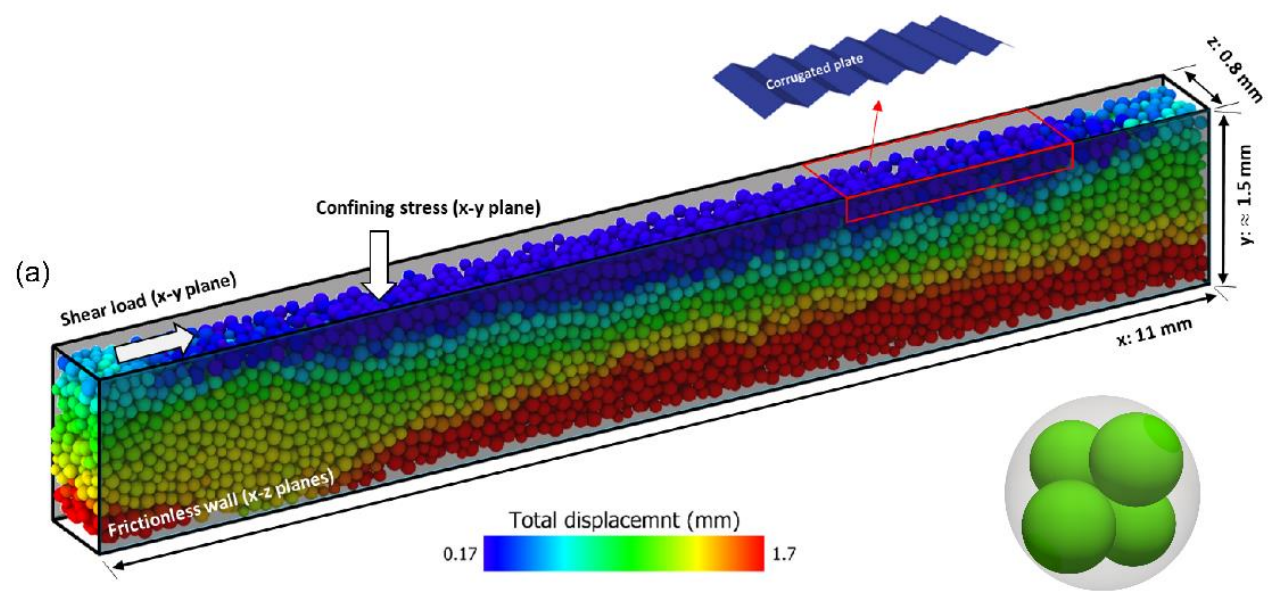

(b) Replacement model

(c)

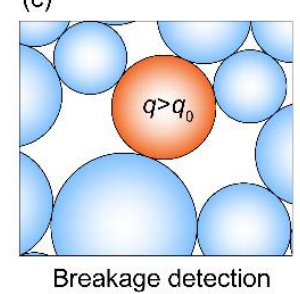

(d)

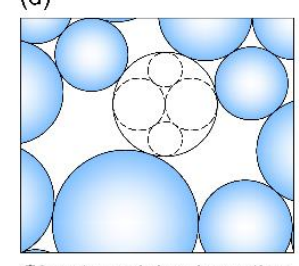

Ghost particles insertion (e)

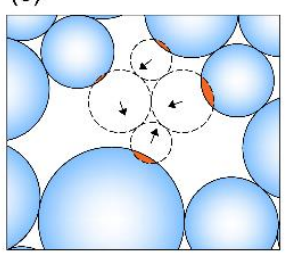

Position adjuestment (f)

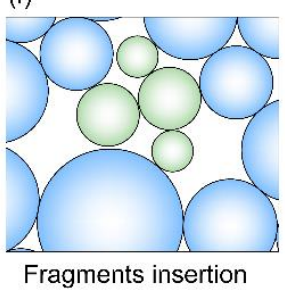

Figure 1: (a) The granular fault gouge consists of 7996 particles. There are two frictionless walls in $x-z$ planes on the front and back of the sample. The fault gouge is confined by two corrugated plates and sheared in the $\mathrm{x}$ direction which allows particles to pass through periodically. (b) Four-particle apollonian sphere model. (c-f) Particles with an octahedral shear stress larger than the strength of 
the grain are replaced with small particles based on apollonian sphere packing. To keep the volume and mass conservation and avoid introducing additional artificial energy by overlap, particles are expanded and rearranged in nearby pores.

\section{3- Results}

Figures 2a-b show the time series of macroscopic friction and thickness of granular gouge comparing the unbreakable (reference run with no fragmentation model) and breakable samples with $q_{\text {crit }}=40 \mathrm{MPa}$. The fragmentation model is inserted at time $0.15 \mathrm{~s}$, where the curves start to diverge and the friction in the model with fragmentation increases. It is obvious that both samples show stick-slip dynamics in the zoom-in panels in Fig. 2c-d: in each stick-slip cycle, the friction increases nonlinearly, reaching a critical state before the slip takes place. From Fig. 2a, it is notable that the friction of the granular fault with particle breakage gradually increases compared to the sample with unbreakable grains. At the end of the simulation, the friction reaches a stable level where the number of fragmented particles keeps decreasing (see the light blue columns in Fig. 2a). This means that due to particle breakage, the fault is strengthening which is consistent with the experimental observations on the spherical beads with particle fracture by Mair et al. (2002). The fragments of beads after breakage have higher surface roughness and angularity, which is represented by a higher inter-particle friction coefficient in our simulations. Therefore, rearrangement and sliding of particles become more limited, and a more stable configuration is reached, leading to a systematic increase in macroscopic frictional strength (Dorostkar and Carmeliet, 2019).

The influence of particle fragmentation is also reflected through the compaction of the granular fault (Fig. 2b). Compaction is a common phenomenon seen in many geomechanical applications that are accompanied by fragmentation events (Fossen et al., 2007; Alikarami et al., 2014; Guo and Morgan, 2007; Mair and Abe, 2011). With increasing number of fragmentation events, more and more smaller particles are generated, which fill the smaller pores, and together with the decrease of particle size cause an overall compaction of the fault gouge. The consistent dilation/compaction pattern for gouge thickness associated with the stick-slip cycles as observed in Fig. $2 \mathrm{~b}$ has been also reported in experiments (Lyu et al., 2019). The stick-slip cycles in Fig. 2c show more frequent drops for the sample with particle breakage compared to the unbreakable one, with many small instabilities in the critical state. The small instabilities recover fast leading to a long stick phase. In fact, most of these minor slip events stem from the local rearrangements due to particle fragmentations and do not directly lead to major slip events. A further discussion of the influence of these particle fragmentation events on stick-slip behaviour will be presented in the discussion section. 
(a)
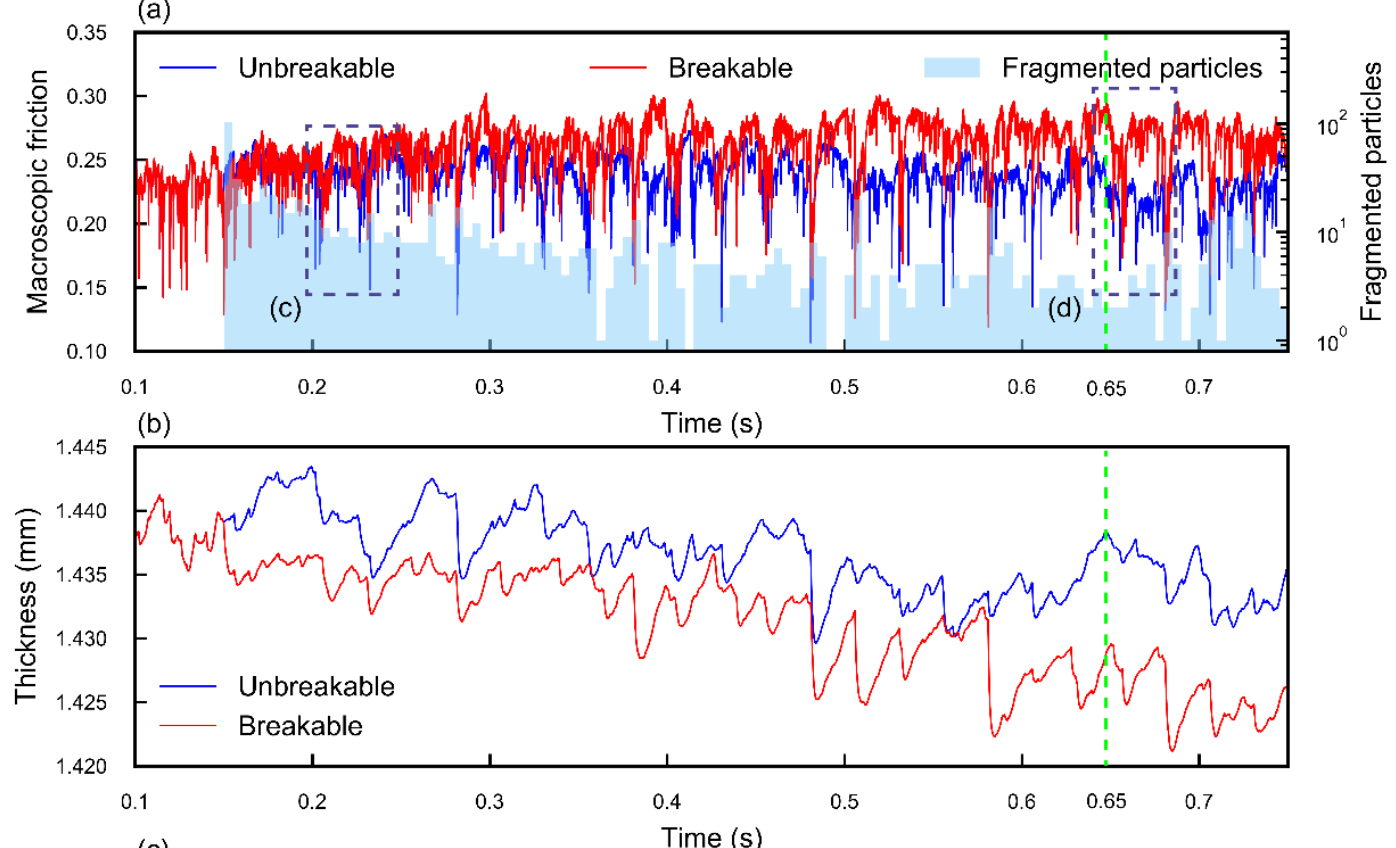

(c)

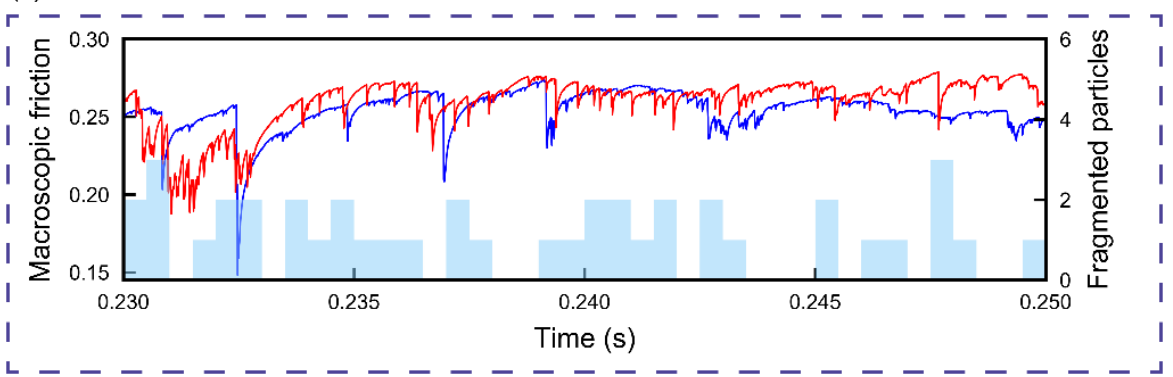

(d)

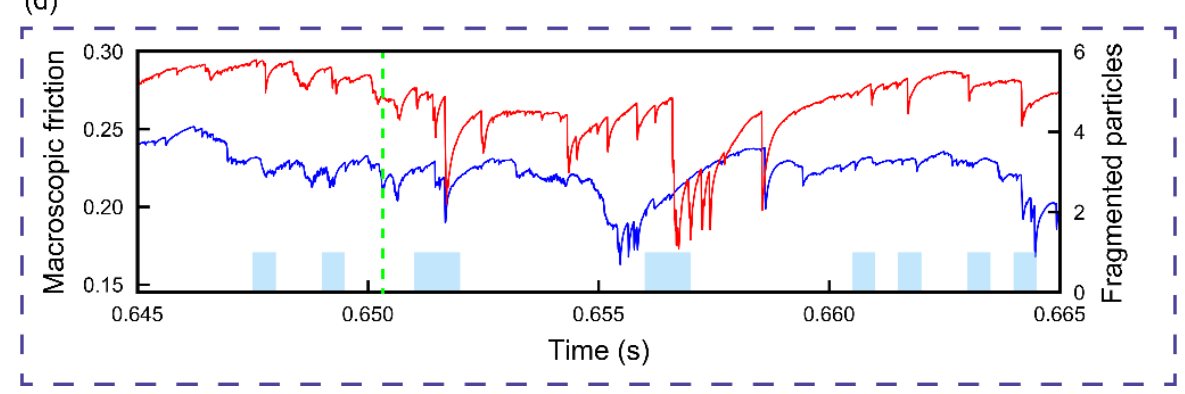

Figure 2: Comparison of friction and thickness between samples with breakable and unbreakable particles: (a) Macroscopic friction, (b) Gouge thickness (y direction in Fig. 1a), (c-d) Marked insets in (a).

After a sufficiently large time of shearing (Fig. 2d), the process of particle fragmentation nearly reaches a steady state value, where the number of broken particles becomes almost constant. At this time, the particle size distribution is approaching an ultimate state (see Fig. 3c). We find that, compared to the sample with unbreakable particles, there are still more small slip events, especially those occurring during the stick phase showing a long term influence of particle fragmentation substantially changing the behaviour of the granular gouge. Continuous particle breakage, the 
reduction of particle size, and generation of high frictional particles contribute to an increase in macroscopic friction (Dorostkar et al., 2019) and number of slip events (Jiang et al., 2016). This combined influence has also been observed in the experiments performed by Mair et al. (2002).

We show in Fig. 3 the evolution of particle size distributions for different critical particle strengths. We also present spatial plots of the particle size distribution at $\mathrm{T}=0.65 \mathrm{~s}$ which is marked with a green dashed line in Fig. 1a. The grain size distribution in the system with particle fragmentation evolves towards a final state that can be described as follows (Turcotte, 1986):

$$
N_{\text {cum }} \propto d^{-D}
$$

where $N_{\text {cum }}$ is the cumulative number of particles and $d$ is particle size and the final fractal dimension $D$ is 2.5 2.7, according to Sammis et al. (1987) and Einav (2007). Here in Fig. 3d the granular assembly attains its final state reaching a grain size distribution with $\mathrm{D}=2.5$. With higher particle strengths, as shown in Fig. 3a-c, the final state is reached more gradually. Observations in Fig. 3 show that our simulations can correctly describe the evolution of fault gouge particle size and capture its transition from a uniform distribution towards a final fractal distribution similar to those observed in experiments (Marone and Scholz, 1989; McDowell, 2002; Baudet, 2018; Zhong et al., 2018).

The evolution towards a fractal type of distribution is controlled by a change in frequency of particle fragmentation events (shown in Fig. 1a). The number of particle fragmentation events at the start of shearing (at small shear strains) is almost an order of magnitude larger than of those events occurring at final state (at larger shear strains), causing a fast evolution of particle size distribution. As time evolves and the total shear strain increases, the number of fragmentation events decreases at decreasing rate, finally converging to fractal PSDs. This evolution stems from the ever-increasing strength of replacing fragments as well as the change in stress state i.e. transition from deviatoric to uniform stress between particles caused by the presence of more small particles (Tsoungui et al., 1999). The former is the size effect as is described in the Weibull type distribution of particle strength, meaning that after particle fragmentation, smaller particles are stronger and harder to be fragmented. On the other hand, when smaller particle fragments fill the fault gouge, the larger particles gain more contacts, which significantly reduces their octahedral shear stress and the fracturing of larger particles becomes more difficult (Zhou et al., 2020). At higher shear strains, as both large and small particles are hard to fragment, the PSD gradually reaches its final state. Fig. 3e-g show the spatial distributions of particle size for different critical particle strengths. In general, with decreasing particle strength, more particles are fragmented and replaced by smaller grains.

We show in Fig. 4a-b, the evolution of mean normal and tangential contact forces for three different critical particle strengths. With decreasing particle strength, the average normal contact force decreases while the average tangential contact force increases. Remark that the average normal contact force is about 8 times higher than the average tangential contact force. The increase of tangential contact force stems from the higher particle friction coefficient of new fragments. On the other hand, the average normal contact force over the whole sample decreases, mainly due to the higher number of contacts created by the small fragments (Fig. 4c). Indeed, Fig. 4c shows a 
significant increase in the number of small contact forces while the strong normal contacts remain unchanged or even increases. The contact force visualization in Fig. 5 shows the overall increase in small contact forces. In addition, the number of relatively strong contacts becomes larger for the sample with breakable particles. This change in normal contact forces is mainly caused as a result of particle fragmentation events, which leads to the particle contact stresses to be redistributed among more particles and over a bigger portion of the sample (de Bono and McDowell, 2016).
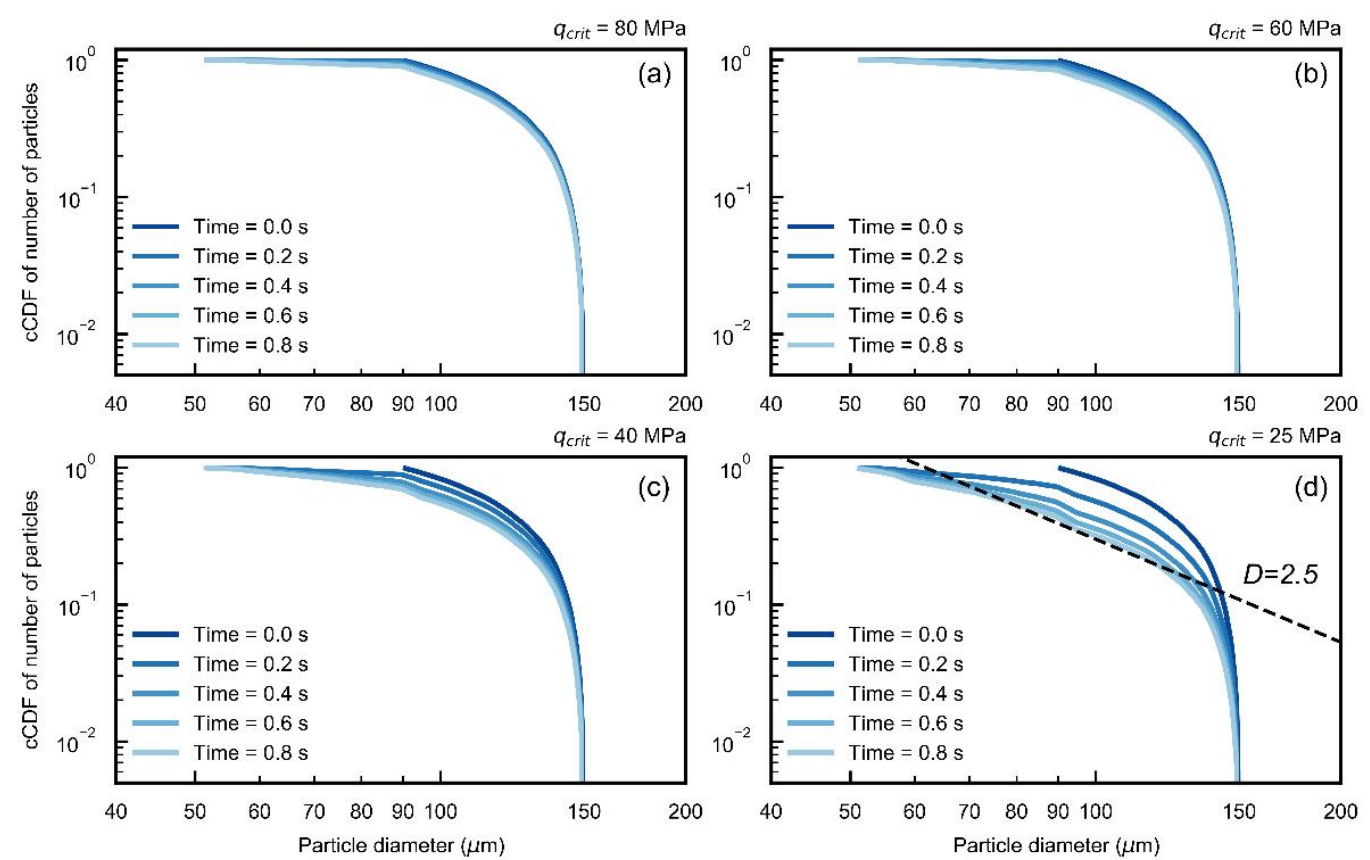

(e)

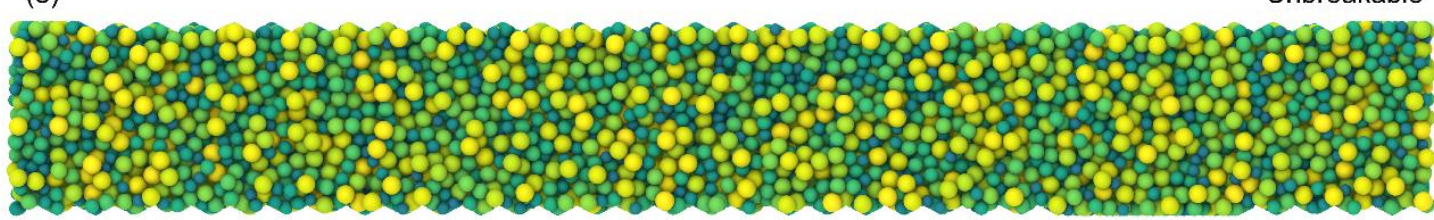

(f)

$q_{\text {crit }}=40 \mathrm{MPa}$

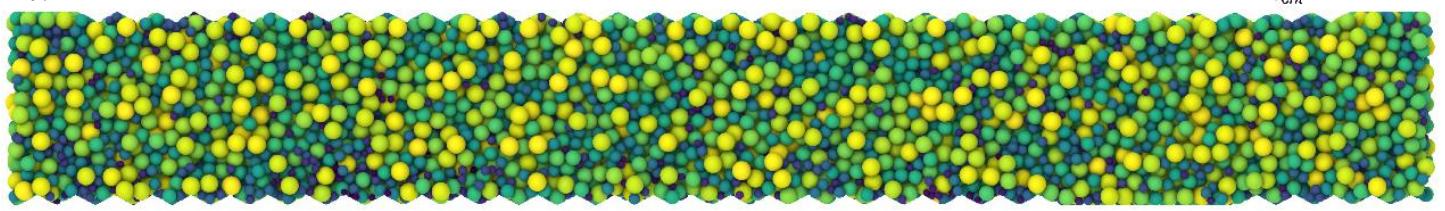

(g)

$q_{\text {crit }}=25 \mathrm{MPa}$
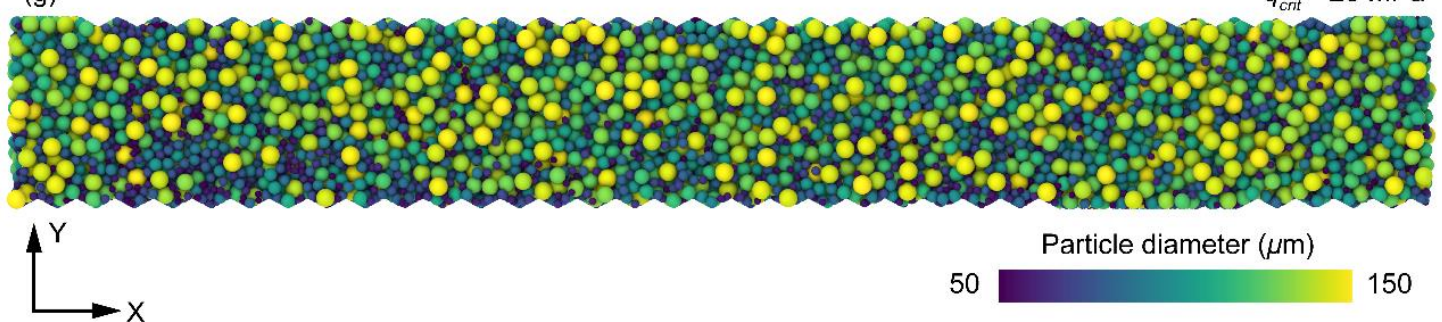

Particle diameter $(\mu \mathrm{m})$

50

150

Figure 3: (a-d) Evolution of particle size distribution and (e-g) spatial distribution of particle size at $\mathrm{T}=0.65$ (s) (marked in Fig. 1a) for simulations with different critical particle strengths. 

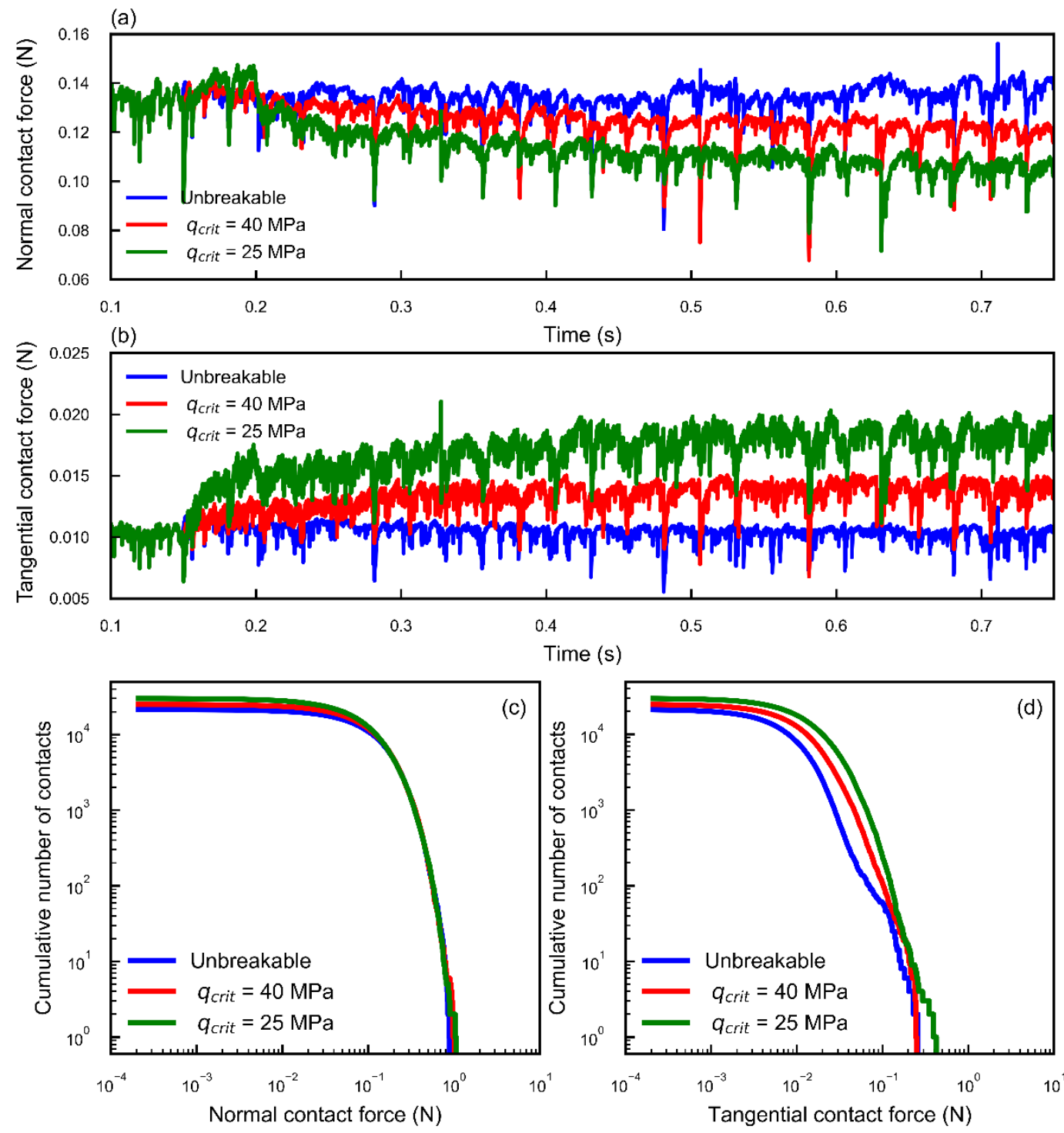

Figure 4: Time series of average (a) normal contact force and (b) tangential contact force. (c-d) The cumulative number of normal and tangential contact forces with different critical particle strengths $(\mathrm{T}=0.65(\mathrm{~s}))$. 

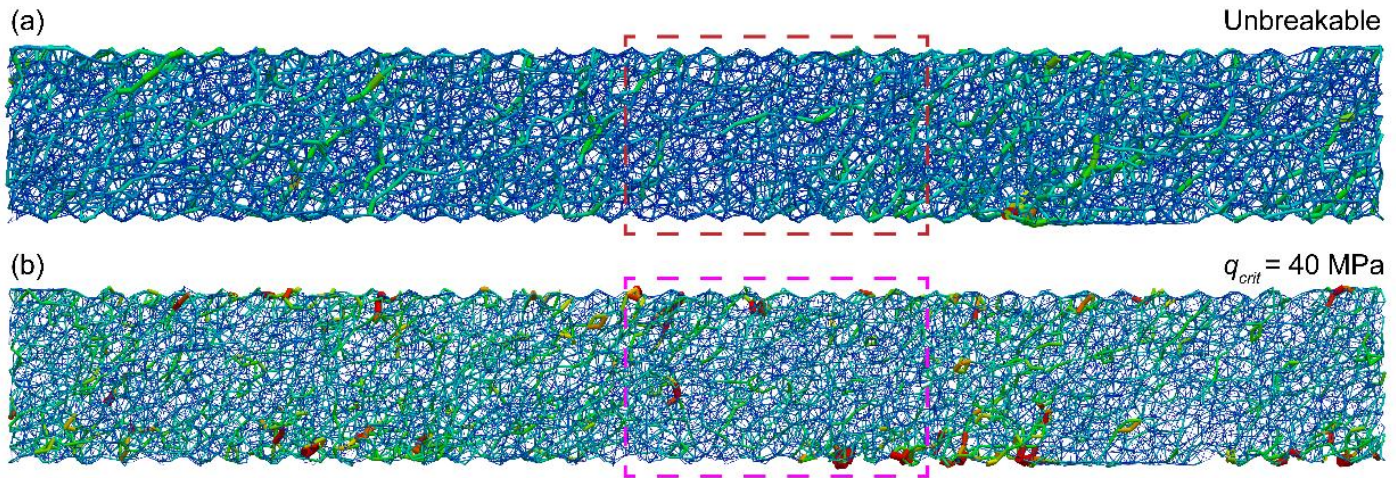

(c)

Unbreakable

(d) $q_{\text {crit }}=40 \mathrm{MPa}$
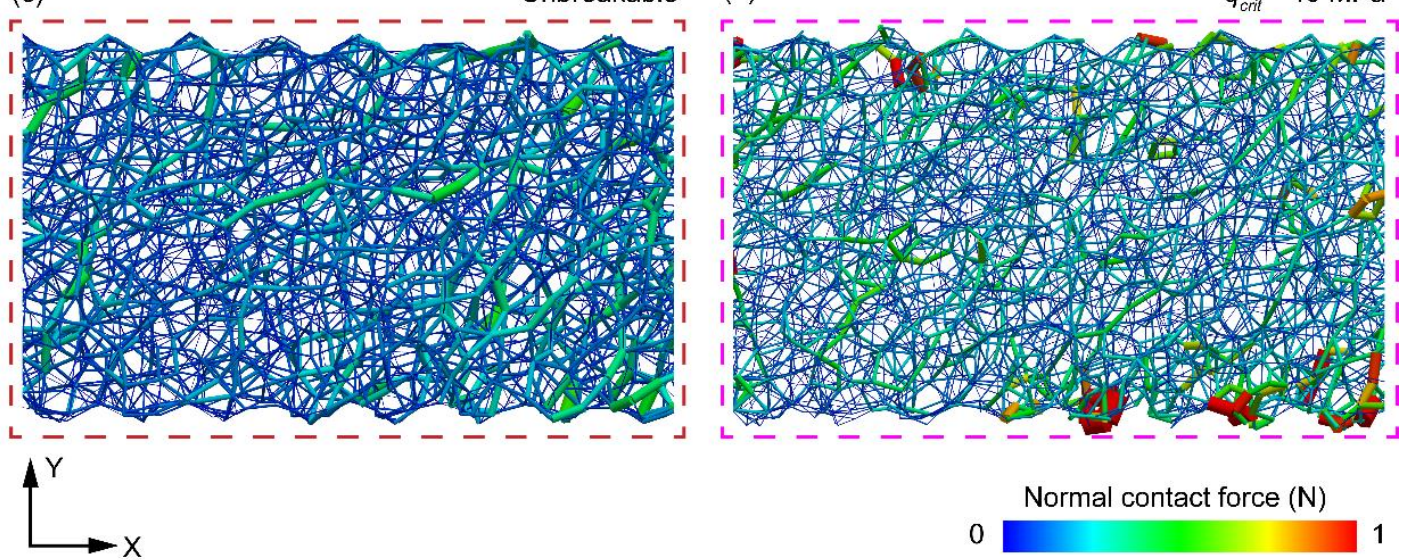

Normal contact force $(\mathrm{N})$

0

Figure 5: Comparison of contact normal forces between samples with (a) unbreakable and (b) breakable particles with $q_{\text {crit }}=40 \mathrm{MPa}$ at $\mathrm{T}=0.65$ (s). (c-d) Marked insets in (a) and (b). The thickness of the lines corresponds to the value of normal contact force, showing more but on average weaker contacts in the sample with fragmentation events. 


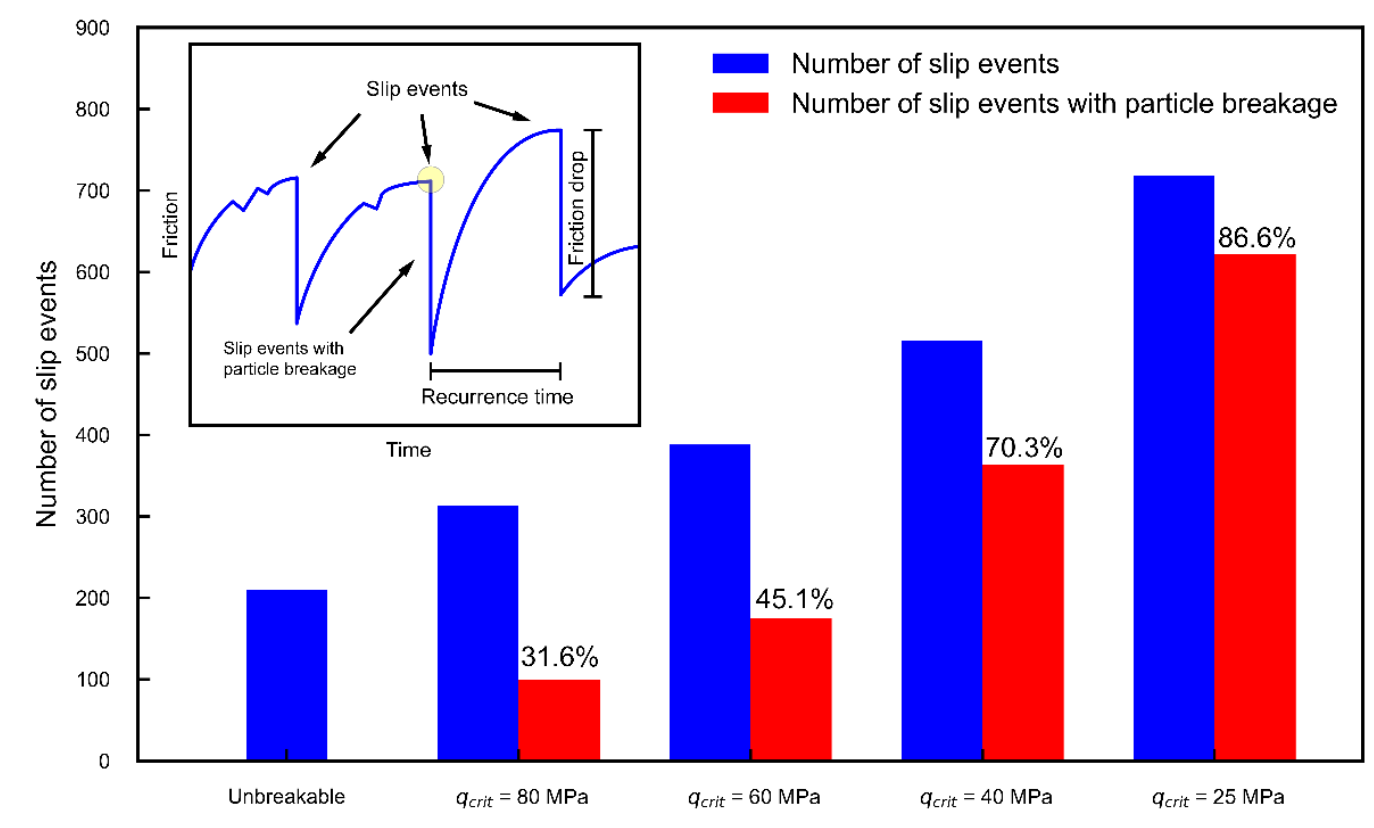

Figure 6: Number of slip events for different critical particle strengths. The blue bars show the total number of slip events, and the red bars show slip events that involve particle breakage. The percentage on the red columns show the proportion of slip events with particle breakage to the total number of slip events. A yellow circle is used to highlight slips that involve particle fragmentation events.

We compare the number of slip events for different critical particle strengths in Fig. 6. The slip events are selected from time 0.2 to 0.75 (s) to avoid the influence of many sudden breakages when initially inserting the fragmentation model. An inset in Fig. 6 schematically presents the definition of slip events, recurrence time and friction drop. For this analysis, slip events with friction drop larger than 0.01 are considered to avoid including micro slips that occur before major slip events mainly due to small rearrangement of particles. Successive small slip events taking place in $50 \mathrm{k}$ time steps are regarded as one slip event with the maximum change of friction taken as friction drop of the major slip event.

In Fig. 6, the blue bars show the total number of slip events while the red bars show those that involve particle breakage. We observe an increase in number of both total slips and slips with fragmentation events with decreasing critical particle strength. This fact demonstrates that particle breakage leads to the occurrence of more slip events, and with decreasing particle strength, this influence is more prominent, meaning that when more particle fragmentation events occur, the correlation between particle breakage and slip events becomes higher. A comparison between the blue and red bars shows that the proportion of slip events with particle breakage increases from $31 \%$ for $q_{\text {crit }}=80 \mathrm{MPa}$ to nearly $87 \%$ at $q_{\text {crit }}=25 \mathrm{MPa}$ (Fig. 6). 


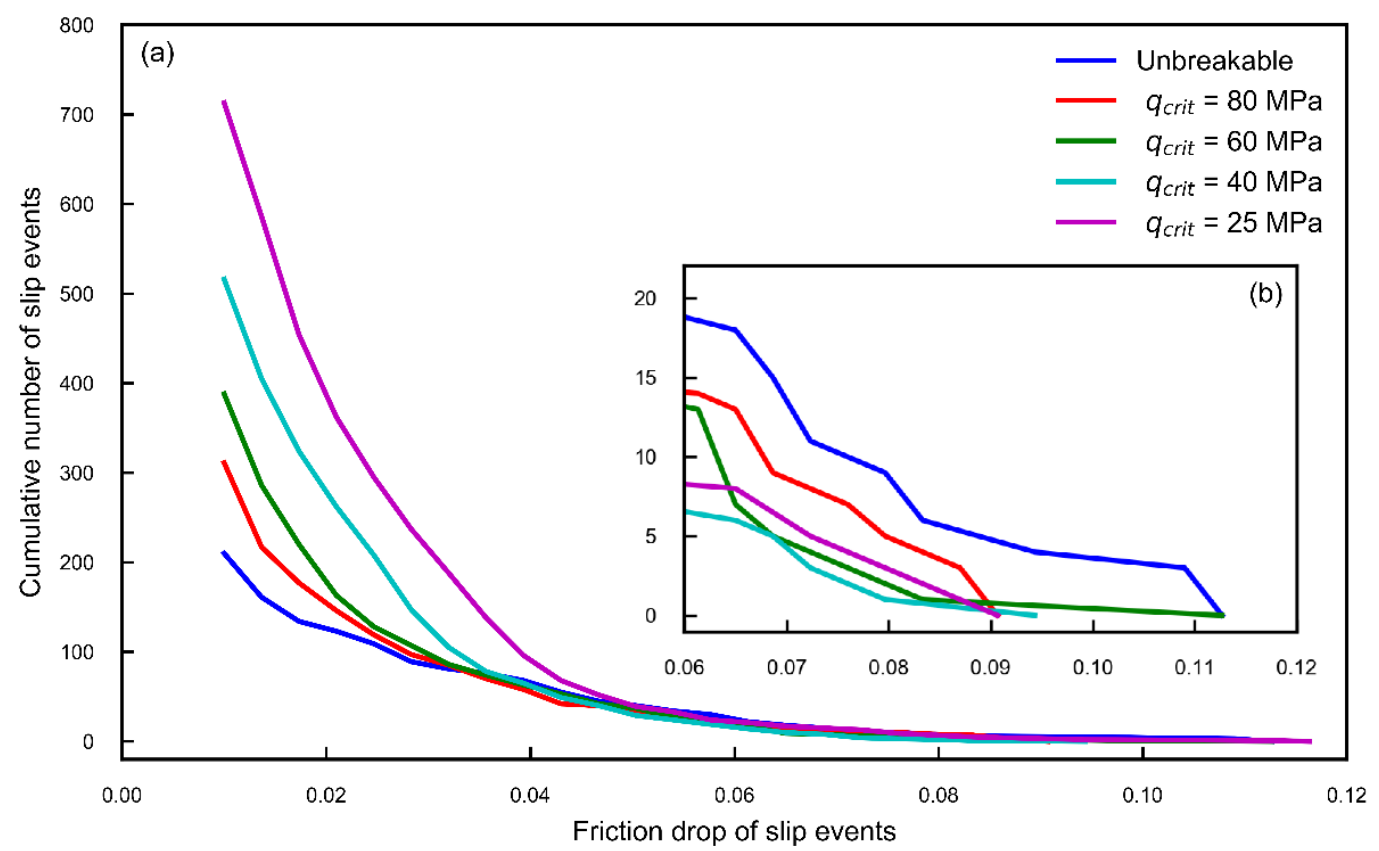

Figure 7: Distribution of slip event size for different critical particle strengths: (a) with the friction drop threshold of 0.01 and (b) with the friction drop threshold is 0.06 .

The distribution of slip events size sheds more light on the increase of number of slip events under different particle strengths. From Fig. 7a, it is clear that the higher number of slip events at lower particle strengths can be attributed mainly to occurrence of smaller slips. The range of this influence also changes with particle strength; for high particle strength where the effect of particle fragmentation is weak, the increase of number of slip events occurs mainly in the range of friction drops smaller than 0.03 , while for lower particle strengths, the increase in number of slips is in the range of friction drops of nearly 0.05 . This demonstrates that particle fragmentation events show a high potential to cause small slips, where as observed in Fig. 2c, most of these small slips take place during the stick phase having little influence on the critical state and slip.

When it comes to the large slip events (friction drop $>0.06$ ), the number of slip events seems to converge (Fig. 7a), since larger events are mostly associated with large particle rearrangements (Dorostkar, 2018), even though particle fragmentation introduces a small disturbance to the stick phase. Nevertheless, Fig. $7 \mathrm{~b}$ shows there is an overturn on the size distribution, where the unbreakable sample has a slightly higher number of large events. This is because, some of the slip events in the unbreakable sample change into a two-stage failure with smaller drops due to the particle fragmentation. Therefore, particle breakage also has an obvious influence on large slip events: they prolong the stick phase by introducing many small slips and occasionally change the major slip event into multi-stage slips.

We also measure the average macroscopic friction, gouge thickness, slip recurrence time and friction drop at major slips for different critical particle strengths (Fig. 8). We observe that the macroscopic friction increases and the gouge thickness decreases as the particles get weaker. Here the lower particle strength leads to a further reduction of particle size and results in many small but rough 
particles, which contribute to the strength of gouge, increasing the friction and causing higher compaction.

We also observe that in systems with weaker particles, the recurrence time gradually decreases. At the same time, as shown in Fig. 8c, with a decrease in particle strength, the standard deviation of the recurrence time also decreases significantly showing that the stick-slip cycles become more regular. In low particle strength samples, the frequency of stick-slip cycles is gradually dominated by the frequent particle fragmentation events and consecutive slips.
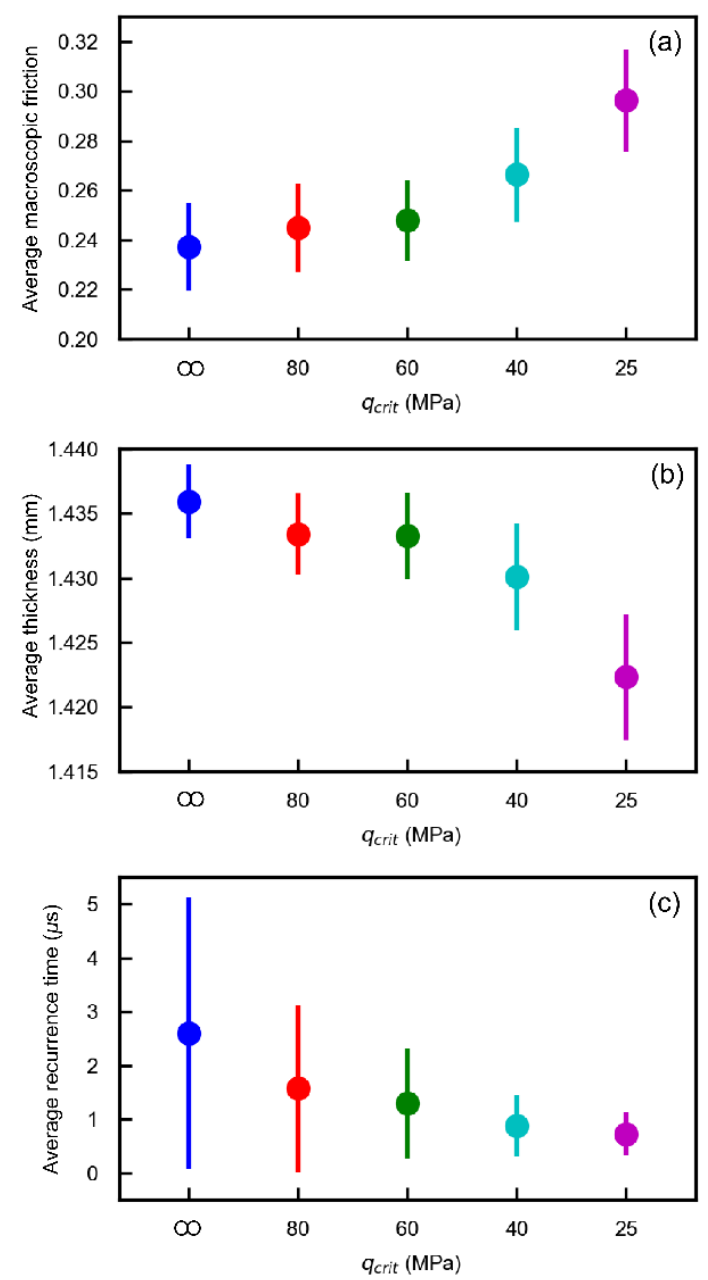

Figure 8: (a) Average macroscopic friction, (b) gouge thickness and (c) slip recurrence time for samples with different particle strengths.

We mentioned that most particle fragmentation events are associated with small slip events. In this section, we focus in more detail on the mechanisms and relationships between particle fragmentation and slip events. Figure 9a shows the time series of macroscopic friction with all particle fragmentation events marked as a circle, where the size of the circle correlates with the number of broken particles. We observe many small particle fragmentation events during the stick phase and larger scale particle fragmentation events (shown with relatively larger circles) at or shortly after slips (see marked points at the lower parts of the curve). We categorize three 
representative types of particle fragmentation events in Fig. 9b-c, which are plots during a shorter time window of Fig. 9a.

\section{Type 1: Small particle fragmentation events triggering microslips}

The particle fragmentation events of type 1 cause small microslips with macroscopic friction drops lower or around the threshold of 0.01 used to detect slip events. It is found that the size of the friction drops increases with decreasing particle strength. Consistent with friction drop, the potential energy drop and the kinetic energy release also have small values. The spatial distribution of potential energy change for microslips triggered by type 1 of particle fragmentation events show a small and localized affected zone (see Fig. 10a). When a particle gets broken and the replacing fragments lose the original contacts of their parents' particle, they move and rearrange, leading to a decrease in their number of contacts and overlap with neighbouring particles. Therefore, with rearrangements and the decrease in the number of contacts or the contact forces, the stored potential energy decreases in a small amount and becomes partly dissipated. However, this local influence is limited to a small area, as it does not change the stress state of particles that are not directly in contact with the fragmented particle.

\section{Type 2: Particle fragmentation events triggering major slips}

The type 2 of particle fragmentations directly trigger major slips (with friction drops larger than threshold of 0.01) showing a profound influence on the friction signal. This type of particle fragmentation events may have nearly the same number of broken particles (one, two or a few particles) as type 1. However, the bigger size of broken particles or their higher stress state make the influence of particle breakage more prominent inducing major slips characterized by a much larger drop in potential energy. The spatial distribution of potential energy change in Fig. 10b shows that, as an example, a series of particles in chain patterns change potential energy during slip. This observation implies that particle fragmentation events of type 2 not only change the particle state around the local breakage area but also lead to an energy release in the surrounding force chains, where a series of rearrangements is triggered.

\section{Type 3: Post-slip large-scale particle fragmentation events}

Type 3 of particle fragmentation events are fundamentally different to type 1 and 2 events (Fig. 9b and 10c) since they do not trigger slips. They appear at the end of a slip or at the beginning of the following stick phase and occur at a relatively large scale (Fig. 9a). In contrast to type 1 and 2 where particle fragmentation triggers micro- or major slips, type 3 of particle fragmentation is a result of the occurrence of a large slip event, after which large particle rearrangements with high particle stress concentrations take place. When a large slip event happens, a large portion of sample undergoes particle rearrangements with stress redistribution, which may lead to high stresses on particles surpassing their particle strength (Fig. 11). In order to capture the particle information and explore the micromechanics, we chose three observation times around a relatively large particle fragmentation event in Fig. 11a, marked as Points A, B and C. Points A and B are chosen before and Point $\mathrm{C}$ after the fragmentation event. We measure how the octahedral shear stress as breakage 
criterion changes at these three points to better understand the underlying mechanisms of type 3 of particle fragmentation events (Fig. 11). While the centre of gouge shows lower stresses at Point A, we observe several highly stressed particles in the highlighted rectangle with dashed line at Point $B$. Note that while the slip event seems almost completed at Point A, the rearrangements and stress redistribution continue, leading to particle stress build up in the highlighted zone in Point B (Fig. 11c). To clearly show this change in particle stress state, we plot the difference in octahedral shear stress between Point B and A in Fig. 11e, where the stress concentration is clearly observable at the centre of the fault gouge. This localization area is exactly where the highly stressed particles fragment between Point B and C, as is shown in Fig. $11 \mathrm{f}-\mathrm{g}$. It is notable that those highly stressed particles form a cluster rather than being aligned in chain patterns. In contrast to the force chains found during continuous shearing, particle fragmentation in clusters is characteristic for post-slip rearrangements introducing local stress concentrations. The kinetic energy signal at this time in Fig. $9 \mathrm{c}$ also shows that at the particle fragmentation event, the gouge is undergoing large-scale particle rearrangements, where nearly most of the particles are moving and rearranging. During these rearrangements, stress is localized leading to a larger scale particle fragmentation event (Fig. 11fg). Moreover, many particles that are weak but outside the direct environment of major particle rearrangements may get involved and crushed. This type of particle fragmentation event is controlled by slip-induced particle rearrangements, and therefore common to take place only when major slip events occur (Fig. 9a). 

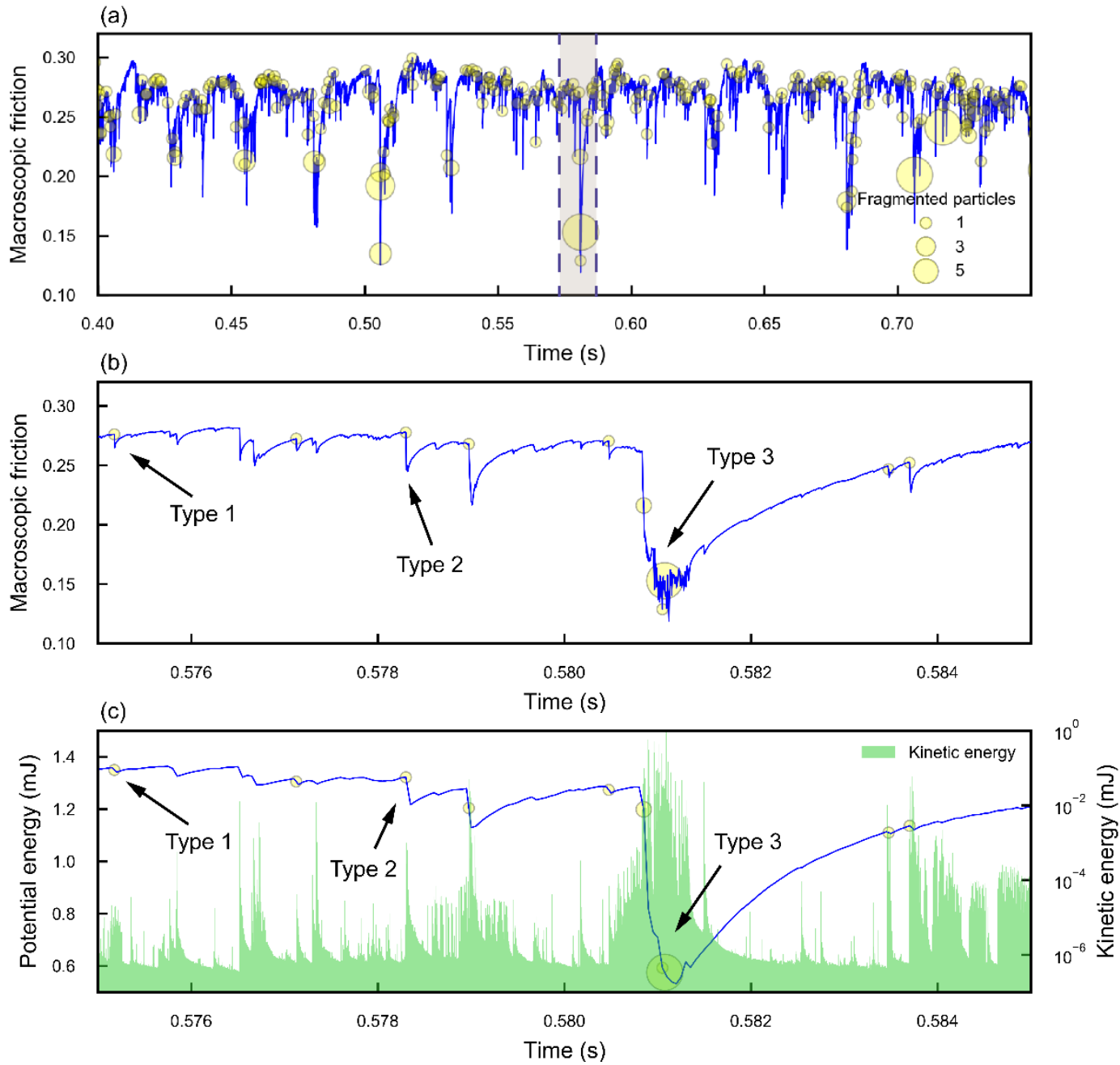

Figure 9: (a) Time series of macroscopic friction under particle strength $q_{\text {crit }}=40 \mathrm{MPa}$ with indication of particle fragmentation events. The yellow circles represent the number of broken particles at each particle fragmentation event. (b) Three types of particle fragmentation events are distinguished showing different relations between particle breakage and slip event in terms of change in macroscopic friction change. (c) Potential and kinetic energy changes versus time indicating three types of particle fragmentation events. 
(a)

Type 1

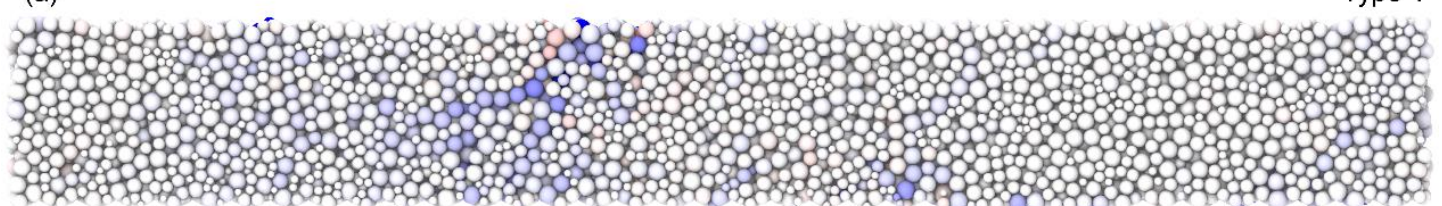

(b)

Type 2

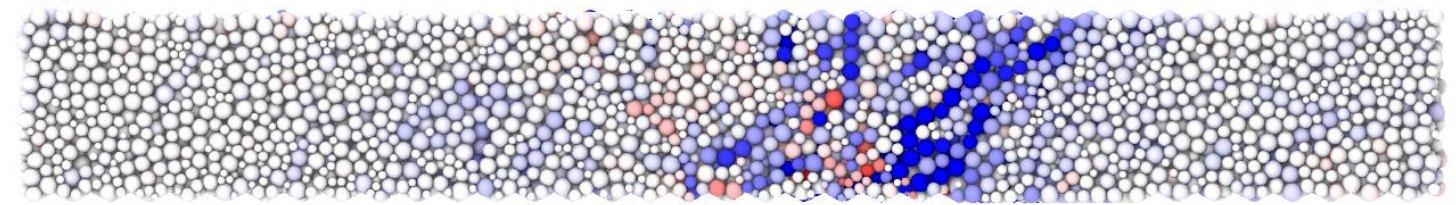

(c)

Type 3

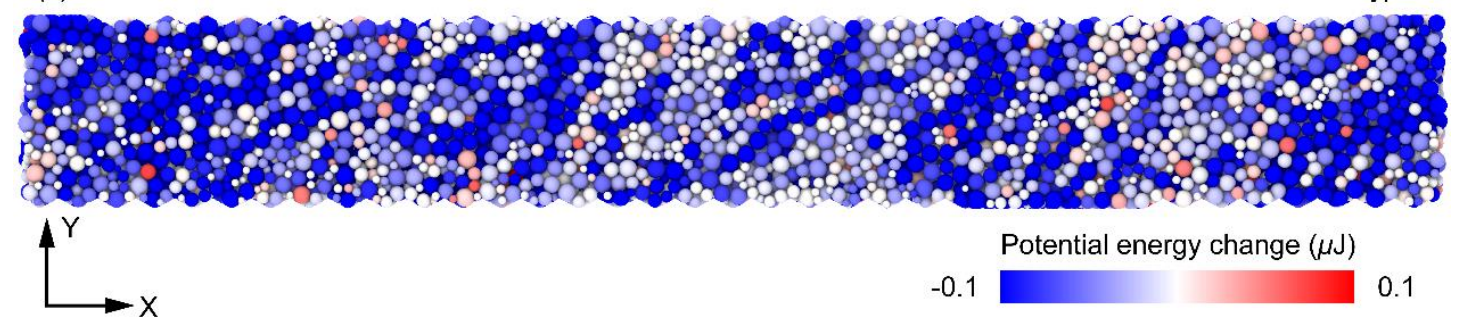

Figure 10: Spatial distribution of potential energy change before and after slip event: (a) type 1, (b) type 2, and (c) type 3 of particle fragmentation events, as marked in Fig. 9b. White particles indicate no or little change in particle potential energy due to the slip. 

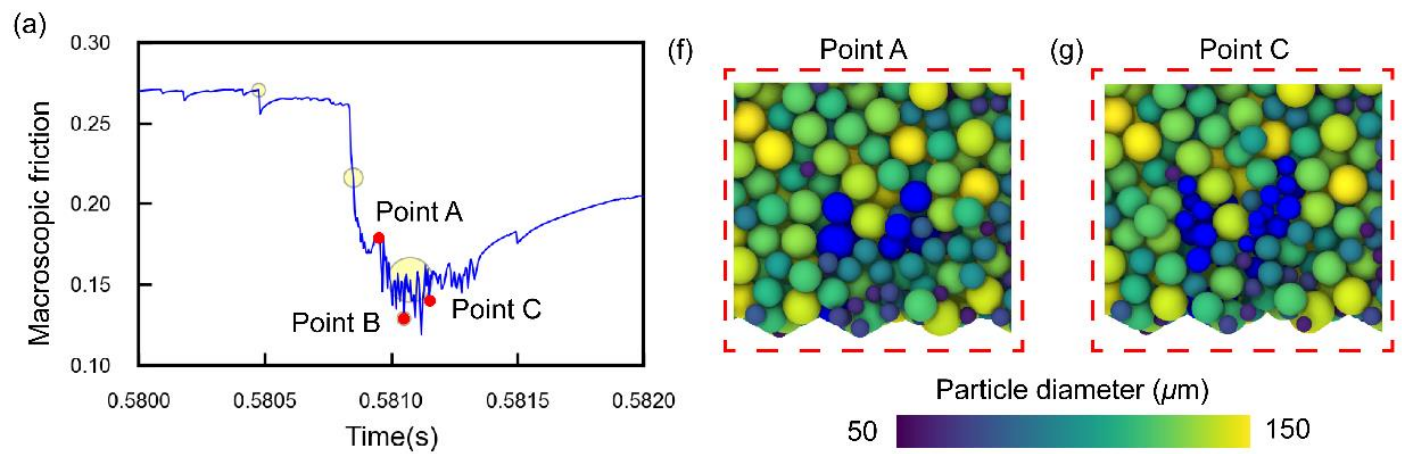

(b) Point $\mathrm{A}$
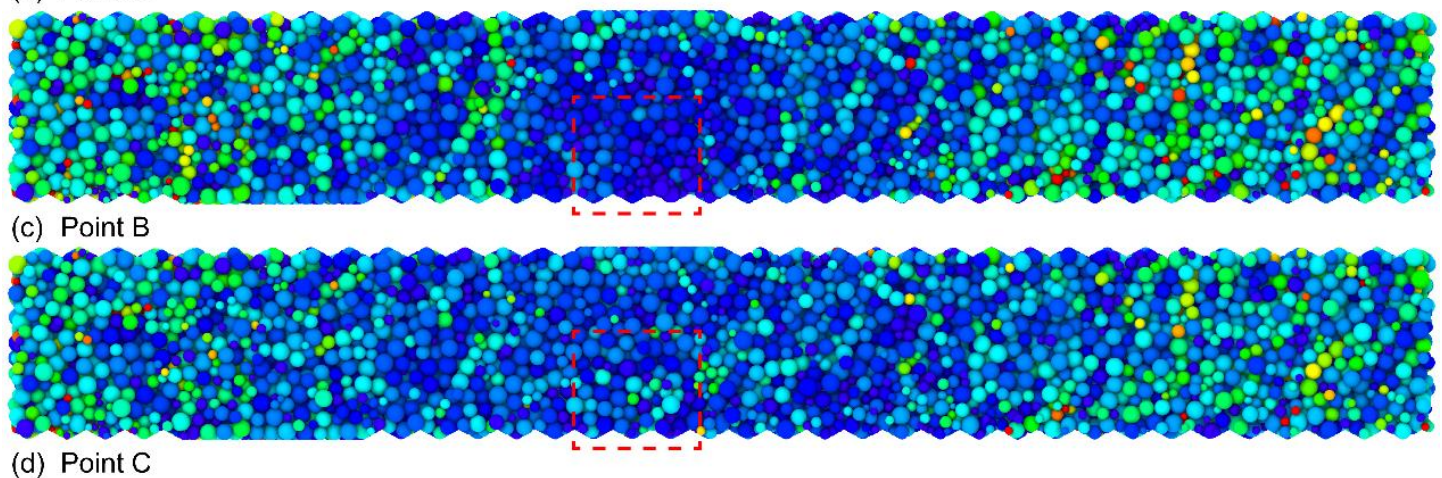

(d) Point C
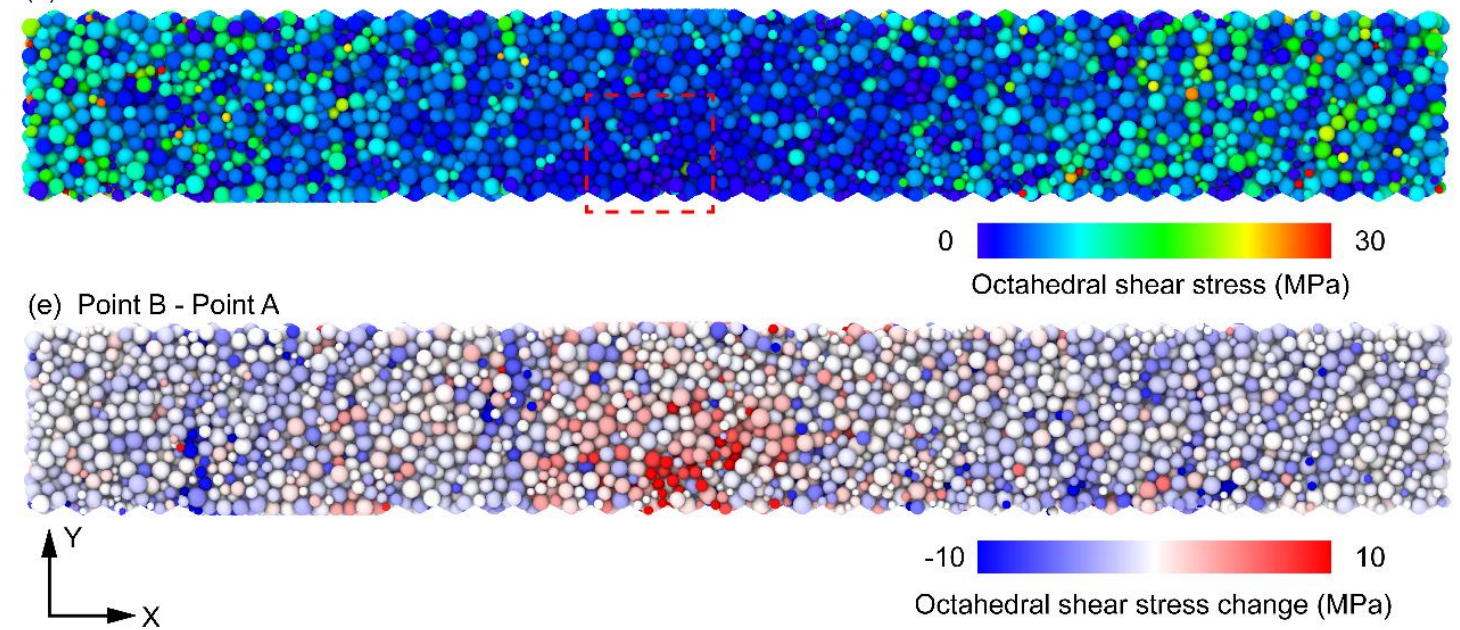

Figure 11: (a) Time series of macroscopic friction near a type 3 fragmentation event as shown in Fig. 9b. Spatial distributions of octahedral shear stress at observation points: (b) stress at Point A, (c) stress at Point B and (d) stress at Point C. (e) Stress variation between Points B and A. (f) Detected particles before fragmentation event (Point $\mathrm{A}$ ) and $(\mathrm{g})$ replacing fragments after the fragmentation event (Point C). Please note that the blue colour of detected particles and replacing fragments does not follow the colour coding for the stress as for visualization purpose. 


\section{4- Discussion}

It may be argued that the distinction between type 1 and type 2 of particle fragmentation events triggering respectively micro- or major slips is based on the choice of a particular threshold for detecting major slips. We however speculate that type 2 of particle fragmentation events may be preceded by type 1 events. In type 1 events, when a particle breaks and gets replaced by smaller fragments, surrounding particles that were previously connected with the fragmented particle may lose part of their contacts leading to a redistribution of potential energy. Due to the limited potential for rearrangements in a dense system like the system we study, surrounding particles may still keep their former stress states and do not fragment. Whether weak zones created by the broken particles in type 1 events can be merged and lead to a type 2 event and a subsequent major slip, is a question that we will address in our future research. It is commonly accepted that particle breakage is highly related to the presence of high stresses on particles forming force chains. It is further recognised that when particles break, particle rearrangements may trigger a major slip event associated with substantial release of potential energy (Mair and Abe, 2008; Davies et al., 2012). This interpretation is consistent with our observations for type 2 of particle fragmentation events, where particle breakage directly triggers major slips. The hypothesis that the occurrence of fragmentation-triggered slips may be preceded by many type 1 small particle breakage events inducing microslips will be analysed in future works. When correct, our argumentation implies that slip or fault failure is hard to be triggered simply by one particle fragmentation event without the accumulation of long-term crushing or damage of grains in granular gouge. In other words, prior to a considerable particle fragmentation-induced major slip or fault failure, there should be many small fragmentation events making the system more susceptible to failure or major slip.

We also discuss that a fragmentation event does not only occur under highly stressed force chain conditions but particle breakage may also occur at the end or after the major slip with relatively lower overall shear stress in the sample. In this kind of particle fragmentation events, several particles are crushed due to the presence of local stress concentrations. This observation is in agreement with observations by Blenkinsop (1991), who compared the fractal dimension of PSDs between San Gabriel fault zone and Cajon Pass core, which had similar conditions of confining pressure, temperature and rock type. Blenkinsop (1991) found that San Gabriel fault shows a higher fractal dimension owing to the more intensive fragmentation due to larger displacements. Our simulation results in this work demonstrate that type 3 of particle fragmentation at the end of major slips due to particle rearrangement and stress localization is an important factor leading to a higher evolution of PSDs.

If we compare the particle breakage mechanisms in types 1,2 with type 3, we can conclude that two major factors are controlling the fragmentation of particles during the stick-slip dynamics of a sheared fault gouge. The first one is the increasing stress and stored energy during the stick phase, leading to breakage of highly stressed particles (type 1 or 2). This mechanism mainly occurs during the stick phase and influences the stability of the fault by disturbing the force chains (remark that we do not present a quantitative definition of force chains in this work), however, since only some particles in high stress force chains break, many other weak particles may survive. The second mechanism playing a role in particle fragmentation at the end of a major slip is dominated by the 
granular rearrangements, which change the stress distribution spatially and introduce high stresses on weak and strong particles leading to the fragmentation of weaker ones (type 3). In other words, since many weak particles are not stressed during the stick phase in the granular system, this type of intensive fragmentation events can occur only after slips, when high stress localization takes place. This type of post-failure particle fragmentation events may have limited influences on the stability of the gouge but have significant contributions to evolution of the PSD and stress redistribution in the fault system.

In this work, we also capture the influence of particle strength on fragmentation events and frictional stability of fault gouge. In a weaker fault gouge, breakage occurs more frequently, leading to more frequent slip events triggered by particle fragmentation. With lower particle strength, the influence of particle fragmentation increases, and larger slips can be triggered by particle breakage. However, the number of slips with a large direct friction drop decreases (see Fig. 7b), indicating that large slip events may be influenced by previous fragmentation-triggered slips which release part of the stored energy in advance. Therefore, a fault system with weaker gouge particles does not necessarily have larger slips, since it can release the stored energy more regularly in time.

\section{5- Summary and conclusions}

We simulate the stick-slip dynamics of a granular fault gouge incorporating particle fragmentation in a discrete element model and study the influence of particle breakage on the characteristics of frictional instabilities. Based on our analyses using statistical features as well as micromechanics of the impact of particle fragmentation, we summarize the main findings of this work as follow:

- Particle fragmentation in a sheared fault gouge leads to reduction of grain size, increase of macroscopic friction, and gouge compaction, which can change the hydro-mechanical properties of fault damage zone.

- Particle breakage leads to the evolution of particle size distribution (PSD), which reaches finally a fractal dimension of 2.5 in our simulations. The evolution of particle size distribution significantly increases the number of weak normal contact forces. As replacing fragments have higher roughness compared to the fragmented particles, the tangential contact forces show an overall increase.

- With decreasing particle strength, the number of slip events increases, where most of the slip events have a relatively small friction drop and behave more like a microslip during the stick phase. However, the small particle fragmentation-induced slips can change the stress distribution and lead to major slips.

- The relationship between slip and particle fragmentation events can be categorized into three types: in the first and second categories, microslips and major slips are triggered by particle fragmentation prior or at slip event, respectively. In a third type of relationship between slip and particle fragmentation, particles break due to high stress concentration owing to large particle rearrangements at the end of large major slips. 


\section{6- Acknowledgments}

Authors thank Chris Marone and Jiaying Liu for helpful discussions. Wang Di acknowledges support from the National Natural Science Foundation of China (Grant No. 51579193, 51609019 and 51825905), the China Scholarship Council (201906270106) and the Science project of China Huaneng Group Co. Ltd (HNKJ18-H26). The data related to this paper can be accessed via an online repository from the link https://doi.org/10.3929/ethz-b-000423980, or by contacting the corresponding authors. The software used for this study is open source and publically available as cited in the main text. 


\section{7- References}

Abe, S., \& Mair, K. (2005). Grain fracture in 3D numerical simulations of granular shear. Geophysical Research Letters, 32(5), 1-4. https://doi.org/10.1029/2004GL022123

Abe, S., \& Mair, K. (2009). Effects of gouge fragment shape on fault friction: New 3D modelling results. Geophysical Research Letters, 36(23), 2-5. https://doi.org/10.1029/2009GL040684

Alikarami, R., Andò, E., Gkiousas-Kapnisis, M., Torabi, A., \& Viggiani, G. (2014). Strain localisation and grain breakage in sand under shearing at high mean stress: insights from in situ Xray tomography. Acta Geotechnica, 10(1), 15-30. https://doi.org/10.1007/s11440-014-0364-6

Baudet, B. A., Han, W., Scaringi, G., Hu, W., \& Zhang, X. (2018). Particle shape factors and fractal dimension after large shear strains in carbonate sand. Géotechnique Letters, 8(1), 73-79. https://doi.org/10.1680/jgele.17.00150

Bergstrom, B. H., Sollenberger, C. L., \& Mitchell Jr, W. (1961). Energy aspects of single particle crushing. Trans. AIME, 220, 367-372.

Biegel, R. L., Sammis, C. G., \& Dieterich, J. H. (1989). The frictional properties of a simulated gouge having a fractal particle distribution. Journal of Structural Geology, 11(7), 827-846. https://doi.org/10.1016/0191-8141(89)90101-6

Billi, A., Salvini, F., \& Storti, F. (2003). The damage zone-fault core transition in carbonate rocks: implications for fault growth, structure and permeability. Journal of Structural Geology, 25(11), 1779-1794. https://doi.org/10.1016/S0191-8141(03)00037-3

Billi, A., \& Storti, F. (2004). Fractal distribution of particle size in carbonate cataclastic rocks from the core of a regional strike-slip fault zone. Tectonophysics, 384(1-4), 115-128. https://doi.org/10.1016/j.tecto.2004.03.015

Billi, A. (2005). Grain size distribution and thickness of breccia and gouge zones from thin $(<1 \mathrm{~m})$ strike-slip fault cores in limestone. Journal of Structural Geology, 27(10), 1823-1837. https://doi.org/10.1016/j.jsg.2005.05.013

Blenkinsop, T. G. (1991). Cataclasis and processes of particle size reduction. Pure and Applied Geophysics, 136(1), 59-86. https://doi.org/10.1007/BF00878888

Ciantia, M. O., Arroyo, M., Calvetti, F., \& Gens, A. (2015). An approach to enhance efficiency of DEM modelling of soils with crushable grains. Geotechnique, 65(2), 91-110. https://doi.org/10.1680/geot.13.P.218

Collettini, C., Niemeijer, A., Viti, C., \& Marone, C. (2009). Fault zone fabric and fault weakness. Nature, 462(7275), 907-910. https://doi.org/10.1038/nature08585

Davies, T. R., \& McSaveney, M. J. (2009). The role of rock fragmentation in the motion of large landslides. Engineering Geology, 109(1-2), 67-79. https://doi.org/10.1016/j.enggeo.2008.11.004

Davies, T. R. H., McSaveney, M. J., \& Boulton, C. J. (2012). Elastic strain energy release from fragmenting grains: Effects on fault rupture. Journal of Structural Geology, 38, 265-277. 
de Bono, J. P., \& McDowell, G. R. (2016). The fractal micro mechanics of normal compression. Computers and Geotechnics, 78, 11-24. https://doi.org/10.1016/j.compgeo.2016.04.018

Dorostkar, O., Guyer, R. A., Johnson, P. A., Marone, C., \& Carmeliet, J. (2017a). On the micromechanics of slip events in sheared, fluid-saturated fault gouge. Geophysical Research Letters, 44(12), 6101-6108. https://doi.org/10.1002/2017GL073768

Dorostkar, O., Guyer, R. A., Johnson, P. A., Marone, C., \& Carmeliet, J. (2017b). On the role of fluids in stick-slip dynamics of saturated granular fault gouge using a coupled computational fluid dynamics-discrete element approach. Journal of Geophysical Research: Solid Earth, 122(5), 36893700. https://doi.org/10.1002/2017JB014099

Dorostkar, O. (2018). Stick-slip dynamics in dry and fluid saturated granular fault gouge investigated by numerical simulations (Doctoral dissertation, ETH Zurich). https://doi.org/10.3929/ethz-b-000283977

Dorostkar, O., Guyer, R. A., Johnson, P. A., Marone, C., \& Carmeliet, J. (2018). Cohesion-Induced Stabilization in Stick-Slip Dynamics of Weakly Wet, Sheared Granular Fault Gouge. Journal of Geophysical Research: Solid Earth, 123(3), 2115-2126. https://doi.org/10.1002/2017JB015171

Dorostkar, O., \& Carmeliet, J. (2018). Potential Energy as Metric for Understanding Stick-Slip Dynamics in Sheared Granular Fault Gouge: A Coupled CFD-DEM Study. Rock Mechanics and Rock Engineering, (McGarr 1999), 1-14. https://doi.org/10.1007/s00603-018-1457-6

Dorostkar, O., \& Carmeliet, J. (2019). Grain Friction Controls Characteristics of Seismic Cycle in Faults With Granular Gouge. Journal of Geophysical Research: Solid Earth, 6475-6489. https://doi.org/10.1029/2019jb017374

Engelder, J. T. (1974). Cataclasis and the Generation of Fault Gouge. Bulletin of the Geological Society of America, 85(10), 1515-1522. https://doi.org/10.1130/00167606(1974) $85<1515:$ CATGOF $>2.0 . C O ; 2$

Einav, I. (2007). Breakage mechanics-Part I: Theory. Journal of the Mechanics and Physics of Solids, 55(6), 1274-1297. https://doi.org/10.1016/j.jmps.2006.11.003

Fossen, H., Schultz, R. A., Shipton, Z. K., \& Mair, K. (2007). Deformation bands in sandstone: a review. Journal of the Geological Society, 164(4), 755-769. https://doi.org/10.1144/0016-76492006$\underline{036}$

Griffith, A. A. (1921). The phenomena of rupture and flow in solids. Philosophical transactions of the royal society of london. Series A, containing papers of a mathematical or physical character, 221(582-593), 163-198. https://doi.org/10.1098/rsta.1921.0006

Guo, Y., \& Morgan, J. K. (2006). The frictional and micromechanical effects of grain comminution in fault gouge from distinct element simulations. Journal of Geophysical Research: Solid Earth, 111(12), 1-21. https://doi.org/10.1029/2005JB004049 
Guo, Y., \& Morgan, J. K. (2007). Fault gouge evolution and its dependence on normal stress and rock strength - Results of discrete element simulations: Gouge zone properties. Journal of Geophysical Research: Solid Earth, 112(10), 1-17. https://doi.org/10.1029/2006JB004524

Jiang, Y., Wang, G., Kamai, T., \& McSaveney, M. J. (2016). Effect of particle size and shear speed on frictional instability in sheared granular materials during large shear displacement. Engineering Geology, 210, 93-102. https://doi.org/10.1016/j.enggeo.2016.06.005

Jiang, Y., Wang, G., \& Kamai, T. (2017). Fast shear behavior of granular materials in ring-shear tests and implications for rapid landslides. Acta Geotechnica, 12(3), 645-655. https://doi.org/10.1007/s11440-016-0508-y

Johnson, P. A., Carmeliet, J., Savage, H. M., Scuderi, M., Carpenter, B. M., Guyer, R. A., et al. (2016). Dynamically triggered slip leading to sustained fault gouge weakening under laboratory shear conditions. Geophysical Research Letters, 43(4), 1559-1565. https://doi.org/10.1002/2015GL067056

Kloss, C., Goniva, C., Hager, A., Amberger, S., \& Pirker, S. (2012). Models, algorithms and validation for opensource DEM and CFD-DEM. Progress in Computational Fluid Dynamics, An International Journal, 12(2/3), 140. https://doi.org/10.1504/PCFD.2012.047457

Leeman, J. R., Saffer, D. M., Scuderi, M. M., \& Marone, C. (2016). Laboratory observations of slow earthquakes and the spectrum of tectonic fault slip modes. Nature Communications, 7. https://doi.org/10.1038/ncomms11104

Lyu, Z., Rivière, J., Yang, Q., \& Marone, C. (2019). On the mechanics of granular shear: The effect of normal stress and layer thickness on stick-slip properties. Tectonophysics, 763(April), 86-99. https://doi.org/10.1016/j.tecto.2019.04.010

Mair, K., Frye, K. M., \& Marone, C. (2002). Influence of grain characteristics on the friction of granular shear zones. Journal of Geophysical Research: Solid Earth, 107(B10), ECV 4-1-ECV 4-9. https://doi.org/10.1029/2001jb000516

Mair, K., \& Abe, S. (2008). 3D numerical simulations of fault gouge evolution during shear: Grain size reduction and strain localization. Earth and Planetary Science Letters, 274(1-2), 72-81. https://doi.org/10.1016/j.eps1.2008.07.010

Mair, K., \& Abe, S. (2011). Breaking Up: Comminution Mechanisms in Sheared Simulated Fault Gouge. Pure and Applied Geophysics, 168(12), 2277-2288. https://doi.org/10.1007/s00024-011$\underline{0266-6}$

Marketos, G., \& Bolton, M. D. (2009). Compaction bands simulated in Discrete Element Models. Journal of Structural Geology, 31(5), 479-490. https://doi.org/10.1016/j.jsg.2009.03.002

Marone, C., \& Scholz, C. H. (1989). Particle-size distribution and microstructures within simulated fault gouge. Journal of Structural Geology, 11(7), 799-814. https://doi.org/10.1016/01918141(89)90099-0

McDowell, G. R., \& Bolton, M. D. (1998). On the micromechanics of crushable aggregates. 
Géotechnique, 48(5), 667-679. https://doi.org/10.1680/geot.1998.48.5.667

Mcdowell, G. R. (2002). On the Yielding and Plastic Compression of Sand. Soils and Foundations, 42(1), 139-145. https://doi.org/10.3208/sandf.42.139

McDowell, G. R., \& de Bono, J. P. (2013). On the micro mechanics of one-dimensional normal compression. Géotechnique, 63(11), 895-908. http://dx.doi.org/10.1680/geot.12.P.041

Nakata, Y., Kato, Y., Hyodo, M., Hyde, A. F., \& Murata, H. (2001). One-dimensional compression behaviour of uniformly graded sand related to single particle crushing strength. Soils and foundations, 41(2), 39-51. https://doi.org/10.3208/sandf.41.2_39

Reches, Z., \& Lockner, D. A. (2010). Fault weakening and earthquake instability by powder lubrication. Nature, 467(7314), 452-455. https://doi.org/10.1038/nature09348

Ren, C. X., Dorostkar, O., Rouet-Leduc, B., Hulbert, C., Strebel, D., Guyer, R. A., et al. (2019). Machine Learning Reveals the State of Intermittent Frictional Dynamics in a Sheared Granular Fault. Geophysical Research Letters, 7395-7403. https://doi.org/10.1029/2019g1082706

Sammis, C., King, G., \& Biegel, R. (1987). The kinematics of gouge deformation. Pure and Applied Geophysics PAGEOPH, 125(5), 777-812. https://doi.org/10.1007/BF00878033

Scholz, C., Molnar, P., \& Johnson, T. (1972). Detailed studies of frictional sliding of granite and implications for the earthquake mechanism. Journal of Geophysical Research, 77(32), 6392-6406. https://doi.org/10.1029/jb077i032p06392

Scholz, C. H. (1998). Earthquakes and friction laws. Nature, 391(6662), 37-42. https://doi.org/10.1038/34097

Scuderi, M. M., Collettini, C., Viti, C., Tinti, E., \& Marone, C. (2017). Evolution of shear fabric in granular fault gouge from stable sliding to stick slip and implications for fault slip mode. GEOLOGY, 45. https://doi.org/10.1130/G39033.1

Storti, F., Billi, A., \& Salvini, F. (2003). Particle size distributions in natural carbonate fault rocks: insights for non-self-similar cataclasis. Earth and Planetary Science Letters, 206(1-2), 173-186. https://doi.org/10.1016/S0012-821X(02)01077-4

Tsoungui, O., Vallet, D., \& Charmet, J. C. (1999). Numerical model of crushing of grains inside two-dimensional granular materials. Powder Technology, 105(1-3), 190-198. https://doi.org/10.1016/S0032-5910(99)00137-0

Turcotte, D. L. (1986). Fractals and fragmentation. Journal of Geophysical Research: Solid Earth, 91(B2), 1921-1926. https://doi.org/10.1029/JB091iB02p01921

Weibull, W. (1951). A statistical distribution function of wide applicability. J Appl Mech, 18, 290293.

Zhong, W., Yue, F., \& Ciancio, A. (2018). Fractal Behavior of Particle Size Distribution in the Rare Earth Tailings Crushing Process under High Stress Condition. Applied Sciences, 8(7), 1058. 
Zhou, W., Yang, L., Ma, G., Chang, X., Cheng, Y., \& Li, D. (2015). Macro-micro responses of crushable granular materials in simulated true triaxial tests. Granular Matter, 17(4), 497-509. https://doi.org/10.1007/s10035-015-0571-3

Zhou, W., Yang, L., Ma, G., Chang, X., Lai, Z., \& Xu, K. (2016). DEM analysis of the size effects on the behavior of crushable granular materials. Granular Matter, 18(3), 1-11. https://doi.org/10.1007/s10035-016-0656-7

Zhou, W., Wang, D., Ma, G., Cao, X., Hu, C., \& Wu, W. (2020). Discrete element modeling of particle breakage considering different fragment replacement modes. Powder Technology, 360, 312-323. https://doi.org/10.1016/j.powtec.2019.10.002 